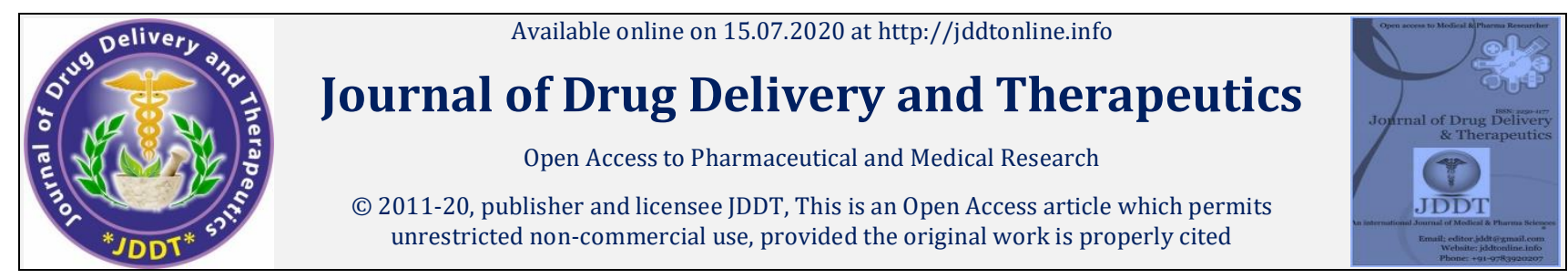

Open 1 Access

Research Article

\title{
Formulation and Physical Characterization of Bio-Degradable Chitosan- Poloxamer Gel Base for Local Drug Delivery
}

\author{
Dahake Prasanna T. ${ }^{*}$, Baliga Sudhindra M. ${ }^{2}$, Punse Triveni ${ }^{3}$, Biyani Dinesh M. ${ }^{3}$, Raut Neha ${ }^{3}$, Umekar Milind J. ${ }^{3}$ \\ ${ }^{1}$ Ph.D. Student, Pharmacology and Pedodontics, (Interdisciplinary Sciences), Datta Meghe Institute of Medical Sciences (Deemed to be \\ University), Sawangi (M), Wardha, Maharashtra, India. \\ Pin code -442001 . \\ 2 Department of Pedodontics and Preventive Dentistry, Sharad Pawar Dental College and Hospital, Datta Meghe Institute of Medical Sciences \\ (Deemed to be University), Sawangi (M), Wardha, Maharashtra, India. Pin code - 442001. \\ ${ }^{3}$ Department of Pharmaceutics, Smt. Kishoritai Bhoyar College of Pharmacy, Kampti, Nagpur, Mahara shtra, India. Pin code - 441002
}

\begin{abstract}
Objective: Thermo-modulated in-situ hydrogel (TSHG) are formulated routinely utilizing poloxamer for extended drug release. However physical properties of such formulations may have some flaws, which can be rectified using a combination of polymers with bet ter physical properties such as chitosan. The purpose of the present study was to fabricate biodegradable chitosan-poloxamer-based in-situ drug delivery systems and assessment of their physical properties.
\end{abstract}

Methods: The present chitosan-poloxamer gel base was formulated using a two-stage method. Initially, chitosan gel was prepared by dissolving $1 \% \mathrm{w} / \mathrm{w}$ chitosan in glacial acetic acid. The poloxamer gel was prepared using "cold method". The final chitosan-poloxamer gel base was prepared by mixing equal amounts of both solutions and evaluated for physical and mechanical properties.

Result and Discussion: The DSC thermogram demonstrated no obvious interactions among ingredients or micellization temperature. The gelation temperature of the gel was between 27 and $33^{\circ} \mathrm{C}$. The $\mathrm{pH}$ was 7 with slight clarity. The viscosity of the gel ranged from 15.14 to 41.19 pa.s. The gel was syringable between $4-30^{\circ} \mathrm{C}$ and biodegradable under physiological conditions. The mean particle size of the gel under SEM was found in the range of 300-554 $\mathrm{nm}$.

Conclusion: After the evaluation of the formulation, it can be concluded that all the ingredients in the gel showed good compatibility wi th each other, which could form a stable and homogeneous gel with favorable mechanical and physical properties.

Keywords: chitosan, drug delivery system, hydrogels, poloxamer

Article Info: Received 21 April 2020; Review Completed 19 June 2020; Accepted 27 June 2020; Available online 15 July 2020

Cite this article as:

Dahake PT, Baliga SM, Punse T, Biyani DM, Raut N, Umekar MJ, Formulation and Physical Characterization of BioDegradable Chitosan-Poloxamer Gel Base for Local Drug Delivery, Journal of Drug Delivery and Therapeutics. 2020; 10(4):59-66 http://dx.doi.org/10.22270/jddt.v10i4.4216

\section{*Address for Correspondence:}

Dahake Prasanna T., Ph.D. Student, Pharmacology and Pedodontics, (Interdisciplinary Sciences), Datta Meghe Institute of Medical Sciences (Deemed to be University), Sawangi (M), Wardha, Maharashtra, India. Pin code - 442001.

\section{INTRODUCTION}

Inventions, besides technological advancements in biogenic natural or synthetic polymers, have shown few promising results using targeted drug delivery systems. Among the recently proposed materials, linear aliphatic polyesters, polyanhydrides, poly (orthoesters), proteins ${ }^{1}$, polylactic acid, polyglycolic acid, and copolymers have extensive applications in local drug-delivery systems. ${ }^{2}$ The embodiment of microparticulate drug molecules in biodegradable polymers is a suitable alternative to some complex medical and dental procedures. Numerous synthetic microspheres are biocompatible, degradable, and easily

ISSN: 2250-1177 convertible to three-dimensional matrixes of variable structures. ${ }^{2}$ The polysaccharides recommended for such purposes include chitin, chitosan, poloxamer, carboxymethyl cellulose, agar, and alginate which are naturally occurring cationic biopolymer.

Apart from its derivation from crustaceans' shells, and the result of the diacylation of chitin ${ }^{3,4}$; chitosan exhibits several physicochemical properties like blending5, Schiff base formation6, compatibility with other bioactive agents 7 , superior biocompatibility, nontoxicity, hemostatic and antimicrobial properties as well. Chitosan is utilized in a wide variety of medical applications such as in situ gels for 
topical application, wound dressing, tissue engineering, and health-care food. It can enhance fibroblast growth, macrophage activation, and hemostasis secondary to wound sealing properties.7 Poloxamer 407 (P407), also termed as Pluronic F127, is a routinely utilized polymer to formulate thermo-modulated in-situ hydrogel (TSHG) for extended drug release. ${ }^{8}$ It can harbor both hydrophilic and hydrophobic drug molecules. ${ }^{9}$ Hence, such a polymer can be effectively utilized for stabilizing single-dose macromolecular formulations. $\mathrm{P} 407$ is a commercially available member of the poly(ethylene oxide-b-propylene oxide-b-ethylene oxide) triblock copolymer family. ${ }^{10}$ It has been utilized as a controlled release vehicle for many macromolecular drugs with variable molecular weights. The methods to prepare drug-loaded TSHGs relatively is modest, utilizing the mixing of ingredients only and sidestepping farthest settings such as heating, the addition of organic solvents, or chemical crosslinking agents. There are experimental studies that have evaluated the effect of individual polymers as drug delivery systems. ${ }^{11}$ Very few experimental studies have attempted to evaluate poloxamer- modified chitosan gels as biologically acceptable bases to improve retention and harmonize their controlled release domain via in-situ ionotropic gelation.

Consequently, the present study was purposed to formulate a biodegradable chitosan-poloxamer in-situ gel base system for local drug delivery.

\section{MATERIALS AND METHODS}

The present in vitro study was conducted at the Department of Pediatric and Preventive Dentistry in association with the Department of Pharmaceutics after gaining clearance from the Institutional Ethical Committee, letter no. DMIMS(DU)/IEC/2015-16/1744, Dated: 31/12/2015.

Materials used for the formulation of the gel base: All materials and chemicals were used in the present experiment were of analytical grade. The quantity and names of various suppliers are mentioned in Table 1.

Table 1: Ingredients used in the gel formulation

\begin{tabular}{|l|l|l|}
\hline Ingredients & Quantity & Supplier \\
\hline Chitosan & $1 \% \mathrm{w} / \mathrm{w}$ & HiMedia Laboratories, Mumbai, India. \\
Poloxamer 407 & $15 \% \mathrm{w} / \mathrm{w}$ & Sigma-Aldrich Mumbai. \\
Carbopol 940 & $0.5 \% \mathrm{w} / \mathrm{w}$ & Akhil Healthcare Private Limited, Gujarat, India. \\
Triethanolamine & $2 \mathrm{drops} / \mathrm{v}$ & Merck Specialities Pvt. Ltd, Mumbai. \\
Methyl paraben & $0.15 \% \mathrm{w} / \mathrm{w}$ & Sigma Aldrich, Mumbai. \\
Hydrochloric acid & $0.1 \% \mathrm{w} / \mathrm{w}$ & Merck Specialities Pvt. Ltd, Mumbai. \\
Propylene glycol & $10 \% \mathrm{w} / \mathrm{w}$ & Merck Specialities Pvt. Ltd, Mumbai. \\
Polyethylene glycol-200 & $10 \% \mathrm{w} / \mathrm{w}$ & Merck Specialities Pvt. Ltd, Mumbai. \\
Isopropyl alcohol & $10 \% \mathrm{w} / \mathrm{w}$ & Sigma-Aldrich, Mumbai. \\
Glacial acetic acid & $2 \% \mathrm{w} / \mathrm{w}$ & Fisher Scientific India, Pvt. Ltd, Mumbai. \\
\hline
\end{tabular}

\section{Preparation of chitosan-poloxamer-based gels:}

A two-stage formulation was utilized to prepare the chitosan- poloxamer gel base. Initially, chitosan gel was prepared by following the method mentioned in a previously sited literature report. ${ }^{12}$ Chitosan $(1 \% \mathrm{w} / \mathrm{w})$ was dissolved gradually in aqueous glacial acetic acid $(2 \% \mathrm{w} / \mathrm{v})$ continuously with slow stirring until complete dissolution. Carbopol $940(0.5 \% \mathrm{w} / \mathrm{w})$ was precisely measured and thawed slowly in double de-ionized distilled water through continuous magnetic stirrer mixing at $200 \mathrm{rpm}$. Both solutions were further blended with continuous stirring. Once the carbopol chitosan solution was prepared, PEG 200 $(10 \% \mathrm{w} / \mathrm{v})$, propylene glycol $(10 \% \mathrm{w} / \mathrm{v})$, isopropyl alcohol $(10 \% \mathrm{w} / \mathrm{v})$, and 2 drops of triethanolamine were added gradually 12 to achieve a clear solution. To prepare the poloxamer gel, the "cold method" was used as stated by Matthew et al. For this purpose, the precisely measured amount of polymer was poured into a flat bottom flask followed by the addition of distilled water and $0.9 \% \mathrm{NaCl}$ to wet it completely with nonstop magnetic stirrer agitation at $100 \mathrm{rpm}$ for $1 \mathrm{~h}$ at room temperature to give partially dissolved solutions. PEG (10\%) was added to this solution with constant magnetic agitation at $100 \mathrm{rpm}$. This solution was further stored at $4^{\circ} \mathrm{C}$ for $24 \mathrm{~h}$ until the entire polymer was completely dissolved to obtain a clear solution.13 The refrigerated chitosan gel was utilized as a solvent for poloxamer dispersion. The final solution of chitosanpoloxamer was prepared by adding an equal amount of both solutions with a continuous stirring mode of $200 \mathrm{rpm}$ for $1 \mathrm{~h}$. At last, methylparaben was incorporated as a preservative. The gel $\mathrm{pH}$ was adjusted by adding $0.1 \% \mathrm{HCl}(\mathrm{w} / \mathrm{v})$ to 7.0 . The solution thus derived was partially dissolved hence refrigerated again at $4^{\circ} \mathrm{C}$ for $24 \mathrm{~h}$ until a clear solution was achieved. This procedure gave a clear tacky solution at the end of $4^{\circ} \mathrm{C}$.

Study of physical interaction between ingredients by differential scanning calorimetry (DSC) study: 3-5 mg of the gel formulation was loaded in an aluminum pan carefully for calorimetric analysis. The pan was carefully sealed by crimping the lid. An empty crimped aluminum pan was used for standard reference. DSC analysis of the gel was performed on a DSC-25 Mettler Toldo system equipped with a refrigerated cooling system and Stare software apparatus (Mettler Toledo, DSC 823e, Greifensee, Switzerland). The experimental and reference pans were heated at predetermined temperature modules in a nitrogen atmosphere at $10^{\circ} \mathrm{C} / \mathrm{min}$, between 20 and $400^{\circ} \mathrm{C}$. Nitrogen was released at $20 \mathrm{ml} / \mathrm{min}$ flow rate at 2 bars. The scans 
were documented, and heat flow-temperature plots were obtained. ${ }^{14}$ The recorded thermogram was verified with individual excipient thermograms to analyze the compatibility and interaction of the excipients with each other. ${ }^{15}$

Physical appearance and pH: The formulations were assessed through visual examination for clarity in front of black and white backgrounds to categorize them as: very clear $[+++]$, clear $[++]$, and turbid $[+] .{ }^{16,17}$ Before estimating the $\mathrm{pH}$ of the gel formulation, the equipment was standardized in the range of 4-9 using standard buffer solutions. Later $\mathrm{pH}$ of the gel was estimated by plunging the glass electrode entirely into the gel. 16

Measurement of gelation temperature and time: The gelation temperature of the gel was measured utilizing the process designated by Garala et al.18 A volume of 3-4 mL gel was transported in a test tube and sealed with paraffin wax. The assembly was dipped in a water bath maintained at $4{ }^{\circ} \mathrm{C}$. The bath temperature was increased incrementally by $2^{\circ} \mathrm{C}$ per minute for 25 mins (range $4^{\circ} \mathrm{C}-54^{\circ} \mathrm{C}$ ). The gelation temperature of the formulation was noted at the point where the meniscus no longer moved by tilting the test tube by more than $90^{\circ}$ angles. ${ }^{8,19}$ To calculate the gelling time, $5 \mathrm{ml}$ sol maintained at $4^{\circ} \mathrm{C}$ was added to a transparent vial with a magnetic stirrer and placed immediately in a water bath $\left(3^{\circ} \mathrm{C}\right)$. The stirring rate of the bar was set at $50 \mathrm{rpm}$. The time when the sol was converted into the gel and the magnetic bar lost movements due to loss of fluidity was considered as the gelation time. ${ }^{8}$

Measurement of viscosity: To determine the viscosity of the gel, $1 \mathrm{ml}$ of the sample was added to a coaxial cylinder viscometer (RST-CC Rheometer, Brookfield Engineering Labs, Inc. Middleboro, USA) that contained a cone with an angle of $1^{0}$, inverted on a stainless-steel cylinder. The viscosity of the gel was estimated at room temperature (range $25-27^{\circ} \mathrm{C}$ ) with a constant shear rate of 6.5/s at a time interval of $4 \mathrm{~s}$ (range 4-40 s), in ascending and descending manner. ${ }^{8}$

Texture profile analysis (TPA): Texture profile analysis (TPA) was assessed in vitro utilizing a CT3 Texture Analyzer (with Texture-Pro CT Software, Brookfield Engineering Labs, Inc. Middleboro, USA) in TPA mode, as recently portrayed.11,20 Sample (35 g) was added to 50 ml bottles, dodging air incorporation into the sample. The analytical probe $(10 \mathrm{~mm}$ diameter $)$ was twofold trampled into the formulation at a predetermined rate of $0.5 \mathrm{~mm} / \mathrm{s}$ to a specified depth of $4 \mathrm{~mm}$. A delay period of 15 $\mathrm{s}$ was permitted amid two compressions at room temperature (range $25-27{ }^{\circ} \mathrm{C}$ ). From the subsequent force-time plots, the hardness, compressibility, adhesiveness, cohesiveness, stringiness, fracturability, and springiness were estimated. 20

Measurement of Spreadability: The spreadability of the gel was estimated with a CT3 Texture Analyzer (with TexturePro CT Software, Brookfield Engineering Labs, Inc. Middleboro, USA) in TPA mode. For that, a set of matched male and female Perspex cones was utilized. All the procedures were followed as explained in the company manual to set up machine assembly precisely, before testing. A conical analytical probe sample holder $(30 \mathrm{~mm}$ diameter, $60^{\circ}$ ) was filled with the gel using a spatula, and its surface was leveled with a flat knife. The conical probe was enforced into the sample holder at a pretest speed of $1 \mathrm{~mm} / \mathrm{s}$. At a trigger force of $10 \mathrm{~g}$, the probe pierced the gel at $2 \mathrm{~mm} / \mathrm{s}$ test speed for $25 \mathrm{~mm}$ depth. After penetration at a specified depth, the probe was retracted from the sample at $2 \mathrm{~mm} / \mathrm{s}$ posttest speed. The subsequent force-time plot thus derived, was the spreadability of the gel.17

In vitro mucoadhesive strength: The mucoadhesive quality of the gel formulation was assessed in vitro through the force essential to detach it from goat cheek mucosa11, utilizing a CT3 Texture Analyzer (with Texture-Pro CT Software, Brookfield Engineering Labs, Inc. Middleboro, USA). 20 The gel was packed into a $30 \mathrm{~mm}$ diameter tube and centrifuged to remove the entrapped air from it, creating a smooth surface. A fresh piece of goat cheek mucosa was obtained and trimmed to dimensions of $(20 \times 20 \mathrm{~mm})$. It was cleaned with a phosphate buffer solution ( $\mathrm{pH}$ 6.8). The mucosa sheet was secured onto the tissue holder, with the orifice of the lid open to expose a small section of the mucosa in a $500 \mathrm{~mL}$ beaker with simulated saliva at $37 \pm 0.5^{\circ} \mathrm{C}$ through a thermostat. The tissue holder was kept in a beaker with stirring to equilibrate at this temperature for 15 mins. The gel was positioned on the mucosal surface through the holder orifice, followed by lowering of the probe assembly on the gel surface at $0.1 \mathrm{~mm} / \mathrm{s}$ continuous speed and, of $7 \mathrm{~g}$ contact force. After $120 \mathrm{~s}$ of contact, the probe was uplifted with $1.0 \mathrm{~mm} / \mathrm{s}$ speed. The area under the curve (AUC) was considered from the force-distance plot as a function of mucoadhesion. ${ }^{21}$

Measurement of Tensile strength: The mechanical properties of the gel formulation were estimated with a CT3 Texture Analyzer (with Texture-Pro CT Software, Brookfield Engineering Labs, Inc. Middleboro, USA), armed with $7 \mathrm{~g}$ of a load cell. The gel was positioned in a 30 $\mathrm{mm}$ tube after freeing from air bubbles and inadequacies, and a $10 \mathrm{~mm}$ analytical probe was twofold trampled into the gel at a predetermined rate of $0.5 \mathrm{~mm} / \mathrm{s}$ to $4 \mathrm{~mm}$ depth at room temperature. A delay period of $15 \mathrm{~s}$ was allotted for two cycles. The subsequent force-time plots for tensile strength for percentage elongation were measured when the gel broke. ${ }^{22}$

Syringability study: The flowability of the gel was analyzed through their syringeability and injectability by utilizing needles like clinical situations or small lab animals like rats or mice. ${ }^{23}$ Through the procedure of withdrawing $1 \mathrm{ml}$ of the refrigerated gel employing $25 \mathrm{G}(0.5 \mathrm{~mm} \times 19 \mathrm{~mm})$ and $22 \mathrm{G}$ $(0.65 \mathrm{~mm} \times 25 \mathrm{~mm})$ needles, was utilized to evaluate the syringeability of the chitosan-poloxamer gel. Similarly, the gel injectability was estimated by regulating it through the same gauge needles affixed to a standard $1 \mathrm{ml}$ syringe with a Luer-Lok system (DISPOVAN, Faridabad, U.P., India). ${ }^{24}$ The criteria followed were classified as (i) injectable suspension (a free-flowing suspension of fine suspended particles that passes easily through a $25 \mathrm{G}$ needle without noticeable resistance); (ii) injectable gel (a solution that passes through a 25G needle with some resistance and emerges as a stream of coagulated gel); (iii) administrable gels (a solution that is difficult to push through a $25 \mathrm{G}$ needle and emerges in the form of gel droplets rather than a stream); or (iv) semisolid gel (a gel solution that cannot be injected through a 25G needle but passes easily through a $22 \mathrm{G}$ needle, emerging as a thick gel). ${ }^{24}$ The gel was stored at $4-8^{\circ} \mathrm{C}$ before syringeability/injectability testing.

Biodegradability test: The biodegradability of the gel formulation was reviewed as per previously published literature. ${ }^{25,26}$ Momentarily, $100 \mathrm{mg}$ sample was transported and incubated into a glass tube comprising $10 \mathrm{~mL}$ of phosphate buffer solution $(\mathrm{pH}$ 7.0) followed by supplementation with $13 \mathrm{mg} / \mathrm{L}$ lysozyme solutions (like in human serum). The blends were kept at $37^{\circ} \mathrm{C}$ with continuous agitation at $60 \mathrm{rpm}$ for various time intervals (1, $2,4,6,8$, and 10 days). The lysozyme solution was 
replenished every day. Toward the finish point of every degradation period, the sample was gathered from the medium, washed with deionized distilled water, and dried at $50^{\circ} \mathrm{C}$ in a heated vacuum chamber until consistent weight. The test was repeated in triplicate, and the weight loss (\%) was determined by the accompanying equation.

$$
\text { Weight loss } \%=\frac{w_{i}-w_{f}}{w_{f}} \times 100
$$

$W i$ is the initial weight of the sample and $W f$ is the final weight of the sample after degradation.

SEM Analysis of the gel: The gel formulation sample was kept at room temperature for $48 \mathrm{~h}$ in a vacuum desiccator to expel any leftover solvent. After mounting the gel sample formulation on the holding device and Au-Pd sputtering, its morphology was imaged at $15 \mathrm{keV}$ under a scanning electron microscope Zeiss EVO-50XVP, (Carl Zeiss SMT, Inc., Peabody, MA, USA). The structural arrangements of the polymeric contents, their orientation, and average particle diameter were determined based on two distinctive SEM micrographs and in all 3 estimations utilizing an image analysis program. ${ }^{24,25}$

\section{RESULTS AND DISCUSSION}

Preparation of chitosan-poloxamer-based gels: This study aimed to develop a biodegradable chitosan-poloxamer gel base for sustained release using chitosan, carbopol as the primary ingredients. Whereas, $\mathrm{P} 407$ as a polymer additive to achieve high performance in situ thermo-reversible matrix gel. Previous reports have elaborated on the poloxamer gel's restricted ability to dissolve quickly in biological and functional environments. ${ }^{27}$ Chitosan harbors very good mucoadhesive properties and augments the gel strength when added at a proper ratio. In the existing study, polymers were added as a second system based on the investigations and reports from previous literature. ${ }^{28}$

Study of physical interaction between ingredients DSC: DSC (Differential Scanning Calorimetry) was utilized in the pharmaceutical field to establish the identity and purity of solid-state systems. It is also used to detect the interaction of the ingredients used in a formulation, and hence, can be utilized for the selection of suitable and chemically compatible ingredients. The thermogram of the gel with all polymers and other ingredients is presented in Figure 1. Chitosan demonstrated a single, defined endothermic peak onset at $124.54^{\circ} \mathrm{C}$, and the peak was obtained at $157.21^{\circ} \mathrm{C}$. While poloxamer demonstrated an endothermic peak at 53 ${ }^{\circ} \mathrm{C}$, however, characteristic peaks of other excipients were unobserved in this figure. Secondly, there was no evidence of any new peak with any of the individual content, suggesting no evidence of excipients and polymer interactions. In this study, DSC was used to study the effects of chitosan and carbopol on the micellization of poloxamer through the critical micellization temperature of the gel. Both the major ingredients, chitosan, and poloxamer, showed typical and clear endothermic peaks, indicative of micellization. This peak is the result of $\mathrm{P} 407$ hydrophobic polypropylene oxide (PPO) block dehydration, through the passage of their micellization, as mentioned in previous reports. ${ }^{29,30}$ The thermogram obtained from poloxamer shows lower values of $\mathrm{T}_{\text {onset, }} \mathrm{T}_{\text {peak, }}$ and $\mathrm{T}_{\text {endset }}$ compared to chitosan. Carbopol and other additives did not disclose significant peaks or changes in the present thermogram. Similarly, the effects of chitosan and carbopol addition on the micellization of P407 were relatively tangible and did not cause any visible alteration in the critical micellization temperature of any ingredient.

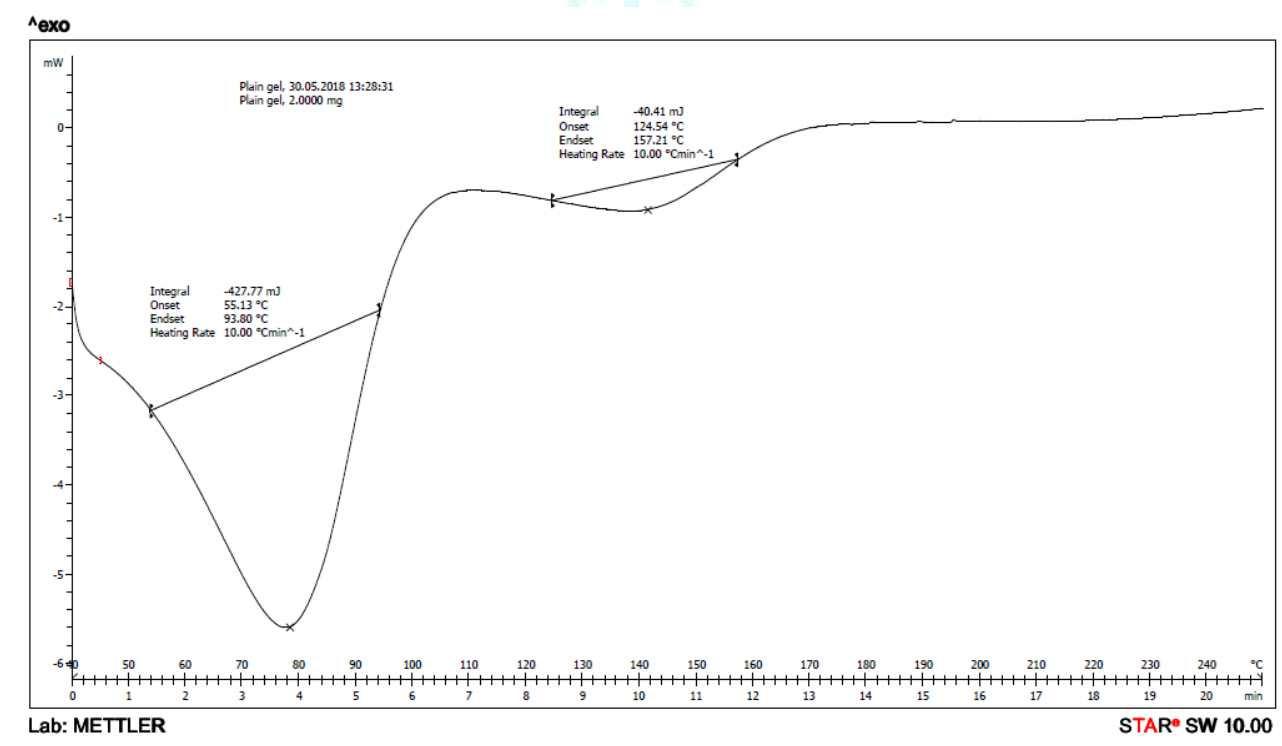

Figure 1: DSC thermogram of the gel formulation

Physical appearance and pH: The physical appearance of the gel was graded clear with a mean value of ++ . The clarity of the gel formulation can be attributed to the non-presence of drug molecules at this phase of a formulation. Likewise, it was not very clear and transparent due to few excipients having different refractive indices, altering the gel clarity slightly. The mean $\mathrm{pH}$ of the present gel was found 7 (with a range of 6.9-7.1) [Table 2].
Table 2: Gelation temperature, time $\mathrm{pH}$, and physical appearance of the gel

\begin{tabular}{|l|l|l|}
\hline Sr. No. & Parameter & Readings \\
\hline 1. & Gelation temperature & $33^{\circ} \mathrm{C}$ \\
2. & Gelation time at $37^{\circ} \mathrm{C}$ & $25 \mathrm{secs}$ \\
3. & pH & 7 \\
4. & Physical appearance & ++ \\
\hline
\end{tabular}


Measurement of gelation temperature and time: The gelation temperature of the gel was between 27 and $33^{\circ} \mathrm{C}$. The gelation time of the present formulation at $37^{\circ} \mathrm{C}$ was found to be $25 \mathrm{~s}$ (Table 2). Certain external triggers alter the gelation temperature and time of such preparations, like a solvent exchange, ionic cross-linkage, $\mathrm{pH}$ change, or UV radiation. The internal triggers that alter gel behavior are the concentration of the ingredients, as mentioned in previous literature. ${ }^{12}$

Measurement of viscosity: In general, an essential factor for an in-situ gel is the gelation temperatures and viscosity whenever applied at the target site for a prolonged stay. Gelation temperature in the range of $25-37^{\circ} \mathrm{C}$ is appropriate as the internal temperature ranges between $36-38^{\circ} \mathrm{C}$. Hence, the study was aimed to prepare an injectable/insertable thermo-reversible in-situ gel system with a gel-sol transition temperature range of $27-33^{\circ} \mathrm{C}$. As presented in Table 3 and Graph 1, the viscosity of the formulation decreased with an increase in shear stress and shear rate. The viscosity of the gel ranged from 15.14 to 41.19 pa.s.

Table 3: Viscosity analysis of the gel formulation at room temperature

\begin{tabular}{|l|l|l|l|l|}
\hline Sr. No. & Time (s) & Shear stress (Pa) & Shear rate (1/s) & Viscosity (Pa.s) \\
\hline 1 & 4 & 00.00 & 0.00 & 0.00 \\
2 & 8 & 264.29 & 6.47 & 40.82 \\
3 & 12 & 324.61 & 12.97 & 25.03 \\
4 & 16 & 363.63 & 19.46 & 18.69 \\
5 & 20 & 395.01 & 25.94 & 15.23 \\
1 & 24 & 392.68 & 25.94 & 15.14 \\
2 & 28 & 357.71 & 19.45 & 18.39 \\
3 & 32 & 319.24 & 12.97 & 24.62 \\
4 & 36 & 267.07 & 06.49 & 41.19 \\
5 & 40 & 42.98 & 0.000 & 00.00 \\
\hline
\end{tabular}

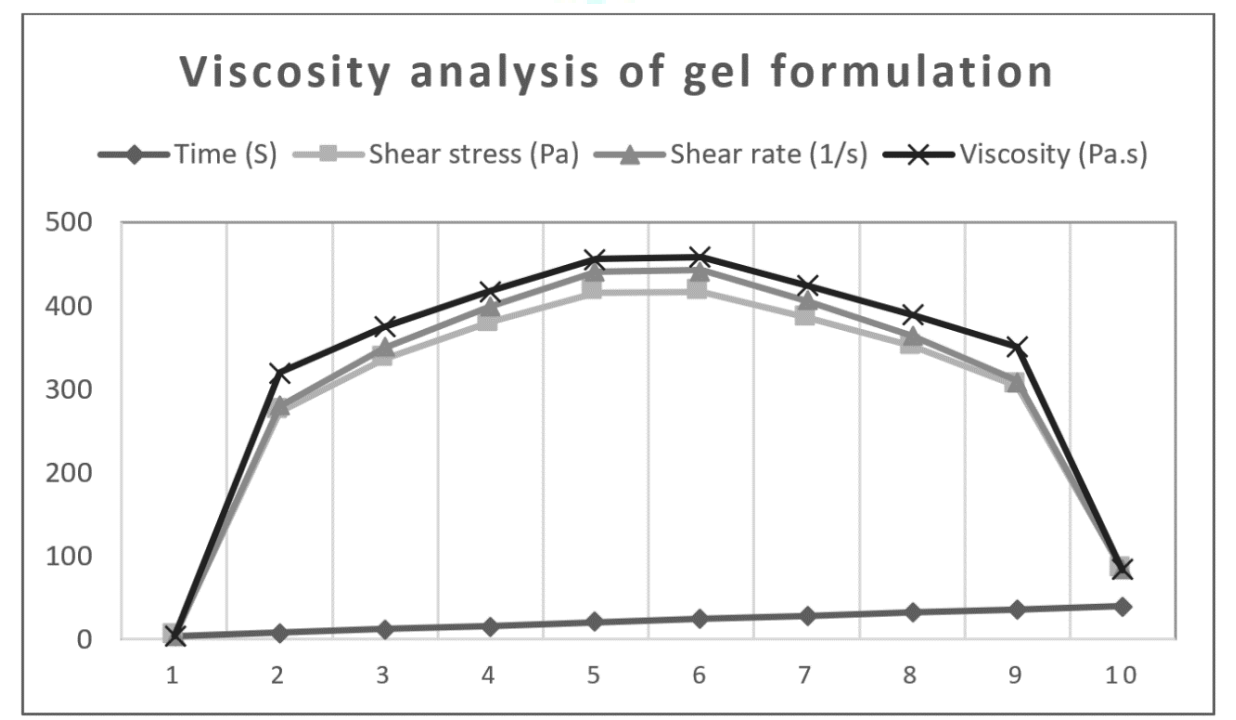

Graph 1: Viscosity analysis of the gel formulation.

Texture profile analysis: Texture profile analysis of the present in-situ gel formulation at a peak load of $32 \mathrm{~g}$ showed hardness up to $3.97 \mathrm{~mm}$, adhesiveness of $0.4 \mathrm{~mJ}$, stringiness of $1.97 \mathrm{~mm}$, fracture deformation at $0.03 \mathrm{~mm}$ ( $7 \mathrm{~g} \mathrm{load})$, the cohesiveness of 0.98 , and springiness of $3.08 \mathrm{~mm}$ (Table 4). These outcomes demonstrated the relevance of gels to target sites or adhesion capacity revealing the retainability of the gel there. Hardness values affirmed the immovability of the gel, while cohesiveness demonstrated better consistency. This will be helpful in cohesion resistance of the formulation in function. 
Table 4: Texture profile analysis (TPA) test method of the gel

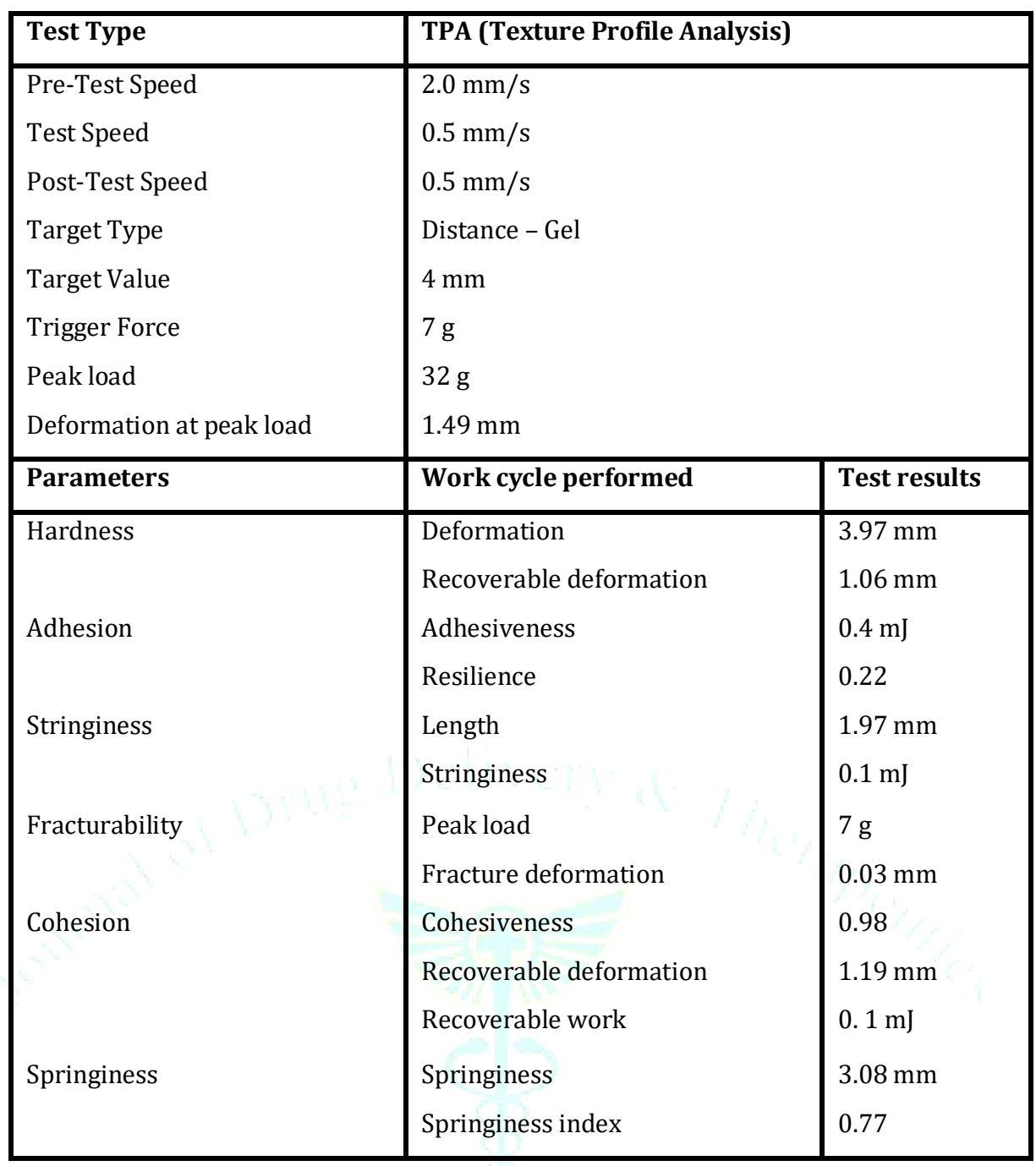

Measurement of Spreadability: Spreadability characterizes the scope of the gel to spread easily during application. Mucoadhesive polymers, by viscosity, demonstrate such properties. Gel spreadability is inversely proportional to its viscosity. Such properties are always desirable for an in-situ gel formulation to deliver active drugs for an ailment. ${ }^{24}$

In vitro mucoadhesive strength: Table 5 shows the mucoadhesion values of the experimental gel. The present gel demonstrated mucoadhesive strength to pick the goat cheek mucosa up to $3.61 \mathrm{~mm}$. This mucoadhesive property is required for retention of the drug-containing gel in situ for better drug release with minimal wastage of the drugs. For the present formulation, mucoadhesion was seen up to 3.61 mm.

Table 5: Mucoadhesion, spreadability, tensile strength of the gel

\begin{tabular}{|l|l|l|l|}
\hline Test Type & Spreadability & Mucoadhesion & Tensile strength \\
\hline Pre-Test Speed & $2.0 \mathrm{~mm} / \mathrm{s}$ & $2.0 \mathrm{~mm} / \mathrm{s}$ & $2.0 \mathrm{~mm} / \mathrm{s}$ \\
Test Speed & $0.5 \mathrm{~mm} / \mathrm{s}$ & $0.01 \mathrm{~mm} / \mathrm{s}$ & $0.5 \mathrm{~mm} / \mathrm{s}$ \\
Post-Test Speed & $0.5 \mathrm{~mm} / \mathrm{s}$ & $4.5 \mathrm{~mm} / \mathrm{s}$ & $0.5 \mathrm{~mm} / \mathrm{s}$ \\
Target Type & Distance - Gel & Distance - Gel & Distance - Gel \\
Target Value & $1.5 \mathrm{~mm}$ & $4 \mathrm{~mm}$ & $1.5 \mathrm{~mm}$ \\
Trigger Force & $13 \mathrm{~g}$ & $7 \mathrm{~g}$ & $7 \mathrm{~g}$ \\
Peak load & $19 \mathrm{~g}$ & $19 \mathrm{~g}$ & $25 \mathrm{~g}$ \\
Deformation at peak load & $1.49 \mathrm{~mm}$ & $3.61 \mathrm{~mm}$ & $3.92 \mathrm{~mm}$ \\
\hline
\end{tabular}


Measurement of tensile strength: Table 5 reveals the tensile strength of the gel formulation. When a peak load of $25 \mathrm{~g}$ was applied to the gel at a speed of $0.5 \mathrm{~mm} / \mathrm{s}$, the gel could bear a tensile strength deformation of up to $3.92 \mathrm{~mm}$. The internal factors modulating the tensile strength of a gel are the percentage distribution of individual composition, adhesion of unlike molecules, and cohesion of like molecules from the ingredients. However, some extrinsic inducements can alter the tensile strength as, the variabilities in force, temperature, and environment. 22

Syringability study: Table 6 reveals the syringeability of the present gel formulation in detail. When present chitosanpoloxamer gel formulation was refrigerated, it maintained its sol form, while at room temperature of $25-27^{\circ} \mathrm{C}$, it lost syringeability within $2-3$ mins. At $25^{\circ} \mathrm{C}$, the gel could be easily withdrawn through a $25 \mathrm{G}$ and $22 \mathrm{G}$ needle. Between $25-27^{\circ} \mathrm{C}$, gel exhibited some resistance to the $25 \mathrm{G}$ needle but extruded easily from the $22 \mathrm{G}$ needle. Hence, the formulation can be considered as "easily injectable suspensions" in refrigerated conditions as a sol, suitable for parenteral application. After $27^{\circ} \mathrm{C}$, the particles started coagulating, requiring the application of little force to inject the solution through the $25 \mathrm{G}$ needle and can be termed as injectable gels. Between $27-30^{\circ} \mathrm{C}$, it was difficult to extrude the gel from the $25 \mathrm{G}$ needle but could be expressed easily through a $22 \mathrm{G}$ needle. Beyond $30^{\circ} \mathrm{C}$, it was difficult to extrude the gel through a $25 \mathrm{G}$ needle, but from a $22 \mathrm{G}$ needle requiring extra pressure and emerged as droplets. Beyond $33^{\circ} \mathrm{C}$ gel did not extrude from the $22 \mathrm{G}$ needle also.

Table 6: Syringeability of the gel formulation at various temperatures

\begin{tabular}{|l|l|l|}
\hline Temperature & Extrusion from 25 G needle & Extrusion from 22 G needle \\
\hline$<25^{\circ} \mathrm{C}$ & Easily - as sol & Easily - as sol \\
$25-27$ & Some resistance - as gel & Easily - as sol \\
$27-30$ & More resistance - as coagulated drop & Some resistance - as gel \\
$30-33$ & Extreme resistance - no extrusion & More resistance as - coagulated drop \\
$>33$ & Extreme resistance - no extrusion & Extreme resistance - no extrusion \\
\hline
\end{tabular}

Biodegradability test: Graph 2 demonstrates the biodegradation profile of the gel formulation, due to the loss of poloxamer, chitosan, and other crosslinking agents due to high water solubility respectively, in the presence of lysozyme. The results revealed that the developed gel was biodegraded due to the liberation of reduced sugar from it. ${ }^{26}$ The degradation rate for the experimental gel gradually increased from $1^{\text {st }}$ to $10^{\text {th }}$ day, ranging from $7 \%$ to more than $60 \%$ of the total volume. However, this property is favorable for the intended use of the gel in situ. The present observation can be justified by the presence of all the hydrophilic water-soluble ingredients, and their high biodegradability. The lysosomal adsorption by this gel is attributed to the presence of several functional hydrophilic $\mathrm{NH}_{2}, \mathrm{OH}$, and $\mathrm{COOH}$ groups in these agents. ${ }^{25,31}$

SEM Analysis of the gel: Figure 2 represents the SEM analysis of the gel formulation. It represents a threedimensional interconnected meshwork of all the ingredients used, without phase separation. The structural arrangements of the polymeric contents look like a blend of polymeric molecules; their orientation is unidirectional, suggesting good adhesion and cohesion of the polymers at $1000 \mathrm{X}$ magnification. The average particle diameter was found to be between 300 and $554 \mathrm{~nm}$.

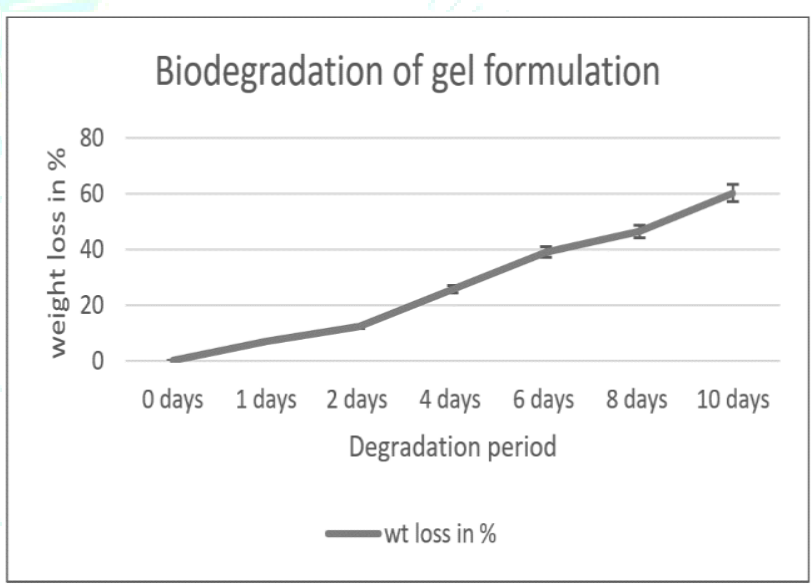

Graph 2 - Biodegradation of the gel formulation in lysosome solution
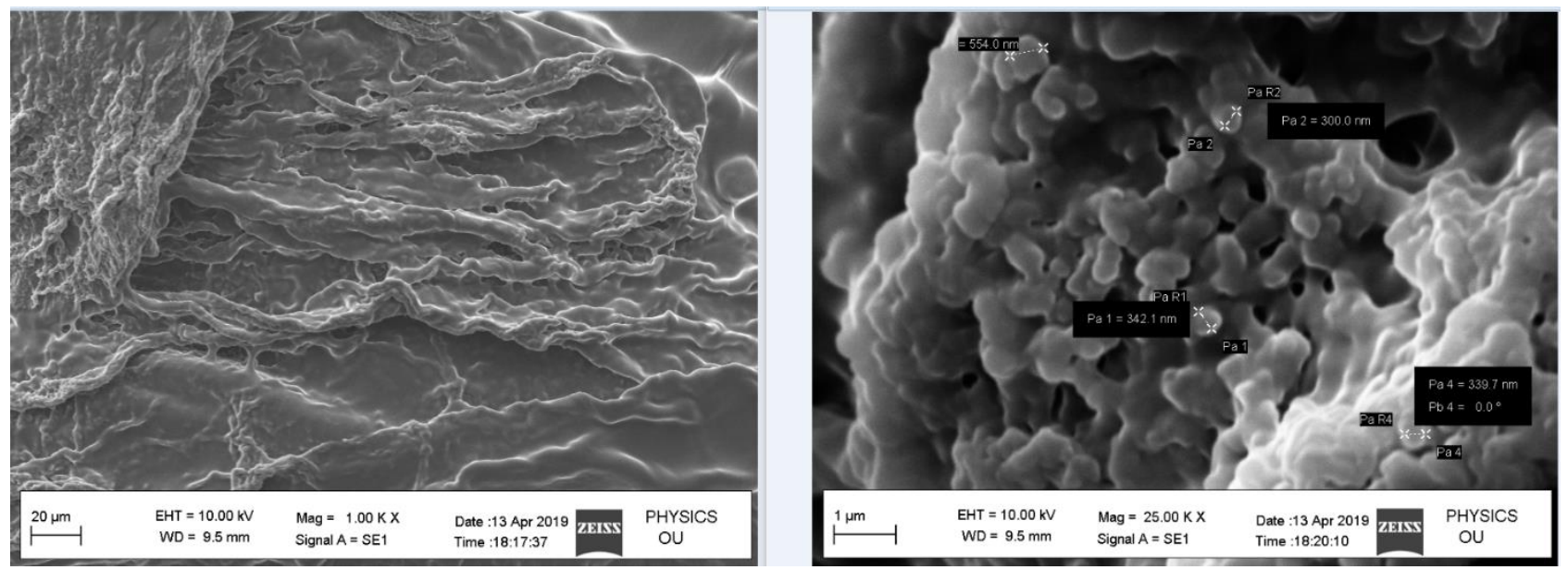

Figure 2: SEM analysis of the gel formulation 


\section{CONCLUSIONS}

After the evaluation of the formulation, it can be concluded that all the ingredients in the gel showed good compatibility with each other, which could form a stable and homogeneous gel with favorable mechanical and physical properties. This biodegradable gel base can be used as a carrier for various drug moieties with prolonged residence time. However, further in vitro and in vivo studies are recommended in the future for bringing this formulation in practical use.

\section{CONFLICT OF INTEREST: Declared none.}

FUNDING SOURCE: This research did not receive any specific grant from funding agencies in the public, commercial, or not-for-profit sectors.

\section{ACKNOWLEDGMENTS: Nil}

\section{REFERENCES}

1. Van de Velde K, Kiekens P. Biopolymers: overview of several properties and consequences on their applications. Polym Test 2002;21(4):433-442.

2. Shive MS, Anderson JM. Biodegradation and biocompatibility of PLA and PLGA microspheres. Adv Drug Deliv Rev 1997;28(1):524.

3. Zargar V, Asghari M, Dashti A. A Review on Chitin and Chitosan Polymers: Structure, Chemistry, Solubility, Derivatives, and Applications. ChemBioEng Rev 2015;2(3):204-226.

4. Rinaudo M. Chitin and chitosan: Properties and applications. Prog Polym Sci 2006;31(7):603-632.

5. Murakami K, Aoki H, Nakamura S, Nakamura S, Takikawa M, Hanzawa M, et al. Hydrogel blends of chitin/chitosan, fucoidan and alginate as healing-impaired wound dressings. Biomaterials 2010;31(1):83-90.

6. Tamer TM, Hassan MA, Omer AM, Baset WMA, Hassan ME, ElShafeey MEA, et al. Synthesis, characterization and antimicrobial evaluation of two aromatic chitosan Schiff base derivatives. Process Biochem 2016;51(10):1721-1730.

7. Tamura H, Furuike T, Nair SV, Jayakumar R. Biomedical applications of chitin hydrogel membranes and scaffolds. Carbohydr Polym 2011;84(2):820-824.

8. Zhang K, Shi X, Lin X, Yao C, Shen L, Feng Y. Poloxamer-based in situ hydrogels for controlled delivery of hydrophilic macromolecules after intramuscular injection in rats. Drug Deliv 2015;22(3):375-382.

9. Liu Y, Lu W-L, Wang J-C, Zhang X, Zhang H, Wang X-Q, et al. Controlled delivery of recombinant hirudin based on thermosensitive Pluronic $®$ F127 hydrogel for subcutaneous administration: In vitro and in vivo characterization. J Controlled Release 2007;117(3):387-395.

10. Klouda L, Mikos AG. Thermoresponsive hydrogels in biomedical applications. Eur J Pharm Biopharm 2008;68(1):34-45.

11. Gratieri T, Gelfuso GM, Rocha EM, Sarmento VH, de Freitas O, Lopez RFV. A poloxamer/chitosan in situ forming gel with prolonged retention time for ocular delivery. Eur J Pharm Biopharm 2010;75(2):186-193.

12. Varshosaz J, Tabbakhian M, Salmani Z. Designing of a Thermosensitive Chitosan/Poloxamer In Situ Gel for Ocular Delivery of Ciprofloxacin. Open Drug Deliv J 2008;2(1):61-70.

13. Matthew JE, Nazario YL, Roberts SC, Bhatia SR. Effect of mammalian cell culture medium on the gelation properties of Pluronic® F127. Biomaterials 2002;23(23):4615-4619.

14. Mora PC, Cirri M, Mura P. Differential scanning calorimetry as a screening technique in compatibility studies of DHEA extendedrelease formulations. J Pharm Biomed Anal 2006;42(1):3-10.
15. Mura P, Faucci MT, Manderioli A, Bramanti G, Ceccarelli L. Compatibility study between ibuproxam and pharmaceutical excipients using differential scanning calorimetry, hot-stage microscopy and scanning electron microscopy. J Pharm Biomed Anal 1998;18(1-2):151-163.

16. Lakshmi P, Harini K. Design and Optimization of Thermoreversible Nasal in situ Gel of Atomoxetine Hydrochloride Using Taguchi Orthogonal Array Design. Dhaka Univ J Pharm Sci 201922;18(2):183-193.

17. Pandit AP, Pol VV, Kulkarni VS. Xyloglucan Based In Situ Gel of Lidocaine $\mathrm{HCl}$ for the Treatment of Periodontosis. J Pharm 2016;2016:1-9.

18. Garala K, Joshi P, Patel J, Ramkishan A, Shah M. Formulation and evaluation of periodontal in situ gel. Int J Pharm Investig 2013;3(1):29.

19. Choi H-G, Jung J-H, Ryu J-M, Yoon S-J, Oh Y-K, Kim C-K Development of in situ-gelling and mucoadhesive acetaminophen liquid suppository. Int J Pharm 1998;165(1):3344.

20. Bruschi ML, Jones DS, Panzeri H, Gremião MPD, de Freitas O, Lara EHG. Semisolid Systems Containing Propolis for the Treatment of Periodontal Disease: In Vitro Release Kinetics, Syringeability, Rheological, Textural, and Mucoadhesive Properties. J Pharm Sci 2007;96(8):2074-2089.

21. Jones DS, Bruschi ML, de Freitas O, Gremião MPD, Lara EHG, Andrews GP. Rheological, mechanical and mucoadhesive properties of thermoresponsive, bioadhesive binary mixtures composed of poloxamer 407 and carbopol 974P designed as platforms for implantable drug delivery systems for use in the oral cavity. Int J Pharm 2009;372(1-2):49-58.

22. Thakur G, Singh A, Singh I. Formulation and evaluation of transdermal composite films of chitosan-montmorillonite for the delivery of curcumin. Int J Pharm Investig 2016;6(1):23.

23. Baumnas V, Remie R, Hackbarth H, Timmerman A. Experimental procedures. In. Principles of Laboratory Animal Science-A contribution to the humane use and care of animals and to the quality of experimental results. 1st ed. Netherlands: Elsevier Science B.V., Amsterdam,; 2001. 313-333 p.

24. Ur-Rehman T, Tavelin S, Gröbner G. Chitosan in situ gelation for improved drug loading and retention in poloxamer 407 gels. Int J Pharm 2011;409(1-2):19-29.

25. Kenawy E, Omer AM, Tamer TM, Elmeligy MA, Eldin MSM. Fabrication of biodegradable gelatin/chitosan/cinnamaldehyde crosslinked membranes for antibacterial wound dressing applications. Int J Biol Macromol 2019;139:440-448.

26. Kumar P, Dehiya BS, Sindhu A. Comparative study of chitosan and chitosan-gelatin scaffold for tissue engineering. Int Nano Lett 2017;7(4):285-290.

27. Dumortier G, Grossiord JL, Agnely F, Chaumeil JC. A Review of Poloxamer 407 Pharmaceutical and Pharmacological Characteristics. Pharm Res 2006;23(12):2709-2728.

28. Packhaeuser CB, Schnieders J, Oster CG, Kissel T. In situ forming parenteral drug delivery systems: an overview. Eur J Pharm Biopharm 2004;58(2):445-455.

29. Su Y, Wang J, Liu H. Melt, Hydration, and Micellization of the PEO-PPO-PEO Block Copolymer Studied by FTIR Spectroscopy. J Colloid Interface Sci 2002;251(2):417-423.

30. Alexandridis P, Holzwarth JF, Alan Hatton T. A correlation for the estimation of critical micellization concentrations and temperatures of polyols in aqueous solutions. J Am Oil Chem Soc 1995;72(7):823-826.

31. Mahnama H, Dadbin S, Frounchi M, Rajabi S. Preparation of biodegradable gelatin/PVA porous scaffolds for skin regeneration. Artif Cells Nanomedicine Biotechnol 2017; 45(5):928-935. 